\title{
Chemical and mineralogical characterization of elbaites from the Alto Quixaba pegmatite, Seridó province, NE Brazil
}

\author{
ANA C.M. FERREIRA ${ }^{1}$, VALDEREZ P. FERREIRA ${ }^{2}$, \\ DWIGHT R. SOARES ${ }^{3}$ and HUGO S. VILARROEL-LEO ${ }^{2}$ \\ ${ }^{1}$ Centro Gemológico do Nordeste, Departamento de Mineração e Geologia, UFCG, \\ Rua Aprígio Veloso, 882, 58109-170 Campina Grande, PB, Brasil \\ ${ }^{2}$ NEGLABISE, Departamento de Geologia, UFPE, \\ Avenida Acadêmico Hélio Ramos, s/n - Cidade Universitária, 50740-530 Recife, PE, Brasil \\ ${ }^{3}$ Departamento de Engenharia de Minas, UFPE, \\ Avenida Acadêmico Hélio Ramos, s/n - Cidade Universitária, 50740-530 Recife, PE, Brasil
}

Manuscript received on August 13, 2004; accepted for publication on April 1, 2005; contributed by VALDEREZ P. FerReIRA*

\begin{abstract}
The Alto Quixaba pegmatite, Seridó region, northeastern Brazil, is a $60^{\circ} / 80^{\circ} \mathrm{SW}$-trending subvertical dike discordantly intruded into biotite schists of the Upper Neoproterozoic Seridó Formation. It has three distinct mineralogical and textural zones, besides a replacement body that cuts the pegmatite at its central portion and in which occur, among other gem minerals, colored elbaites. Elbaites usually occur as prismatic crystals, elongate according to the c-axis, with rounded faces and striations parallel to this axis. Optically, crystals are uniaxial negative with strong pleochroism; refractive index extraordinary axis $=1.619-1.622$ and ordinary axis $=1.639-1.643$, birefringence between 0.019 and 0.021 , average relative density of 3.07 , and the following unit cell parameters: $a \mathrm{o}=15.845 \AA, c \mathrm{o}=7.085 \AA$ and $\mathrm{V}=1540.476 \AA$. There is alkali deficiency in the $\mathrm{X}$ site of $12-17 \%$. The elbaites are relatively enriched in $\mathrm{MnO}$ (1.69 to $2.87 \%$ ) and $\mathrm{ZnO}$ (up to $2.98 \%$ ).
\end{abstract}

Key words: elbaite, tourmaline, Seridó pegmatitic province.

\section{INTRODUCTION}

Numerous occurrences of elbaite-bearing pegmatites in the Borborema pegmatitic province, northeastern Brazil, have been reported in the last years. The elbaites are found as blue, violet-blue, greenish blue, green and dark green crystals, as well as rare bi- and tricolored zoned crystals, in which cores are deep pink, characteristics that make the Seridó region to become worldwide known as producer of rare gem-quality tourmalines. In spite of this, only few studies on the crystal chemistry of

\footnotetext{
*Member, Academia Brasileira de Ciências

Correspondence to: Ana Cláudia Mousinho Ferreira

E-mail: a.claudia1@ig.com.br
}

tourmalines of the many pegmatites in the region have been carried out.

One important characteristic of these elbaites is their high $\mathrm{Cu}$ contents that are responsible for the hues of blue and green colors, such as those from the Boqueirãozinho and São José da Batalha pegmatites studied by Brandstaetter and Niedermayr (1994) and Rossman et al. (1991).

The objective of this study is to present some of the crystallographic, chemical, and gemological aspects of elbaites from the Alto Quixaba pegmatite, and make some inferences about the origin of tourmaline color. 


\section{GEOLOGICAL FRAMEWORK}

The studied pegmatite occurs in the Borborema pegmatitic province, part of the Neoproterozoic Seridó tectonostratigraphic terrane, in the Borborema structural province (Almeida et al. 1981). The Seridó terrane is mostly made up of polideformed Neoproterozoic Seridó Group metasedimentary rocks, and discordantly overlies rocks of the Caicó Complex basement and is constituted, from base to top, by the Jucurutu, Equador and Seridó Formations. These sedimentary sequences, metamorphosed in the amphibolite facies, are intruded by a large volume of plutonic, mostly granitic, Neoproterozoic rocks, during the Brasiliano orogenic cycle.

The Borborema pegmatitic province (Paiva 1946), at the eastern and southeastern part of the Seridó terrane, is characterized by a large number (around 1500 occurrences; Silva 1995) of heterogeneous, both simple and complex pegmatite plutons dated between 450 and 510 Ma. Pegmatites lacking economically important minerals consist of $\mathrm{K}$ feldspar, albite, quartz, and muscovite, while mineralized pegmatites contain $\mathrm{Nb}, \mathrm{Ta}, \mathrm{Sn}, \mathrm{Be}, \mathrm{Li}, \mathrm{P}, \mathrm{U}$, and Th-rich minerals, and carry many varieties of quartz and gem-quality minerals. Two major areas of occurrence are described in the province; one of them encompasses beryl-bearing pegmatites and the other one, tourmaline-bearing pegmatites. The Alto Quixaba pegmatite is one of the tourmaline-bearing pegmatites, intrusive into biotite schists of the Seridó Formation, occurring at about $15 \mathrm{~km} \mathrm{NE}$ of the Frei Martinho town, north of the State of Paraíba, near the boundary with the State of Rio Grande do Norte (Fig. 1).

\section{THE ALTO QUIXABA PEGMATITE}

The Alto Quixaba pegmatite is a typical zoned granitic pegmatitic body enriched in lithophile elements, composed of two distinct bodies that are perpendicularly intercrossed. One of them, of major economic importance, is about $15 \mathrm{~m}$ long and $6 \mathrm{~m}$ of average width. It is $\mathrm{N} 60^{\circ} \mathrm{W}$ oriented, with average dip of $80^{\circ} \mathrm{SW}$, discordant in relation to the
$\mathrm{N} 50^{\circ} \mathrm{E}$-host rocks, which dip $75^{\circ} \mathrm{SE}$ in the local site (Fig. 1).

This pegmatitic pluton presents large mineralogical and textural variations, allowing to the recognition of three main zones (Fig. 2): (a) an external zone composed of K-feldspar, quartz and muscovite, besides black tourmaline that occurs either in massive aggregates or small, brittle crystals of the schorl type; (b) an intermediate zone, a few meters wide, in which microcline, quartz, albite, muscovite, ambligonite and yellowish green beryl occur, besides accessory metallic minerals such as microlite, tantalite to manganotantalite, and blue to greenish blue tourmaline, which usually is opaque, radial, associated with K-feldspar, and less common, with quartz; and (c) an inner zone (nucleus) composed of grayish quartz that, although not well defined, seems to occur as a series of small, narrow and elongate discontinuous segments. The contacts among the three zones are gradational.

There is a subvertical, replacement body intercepting the central part of the pegmatite, a typical replacement body that is usually found in or around complexly-zoned granitic pegmatites. This alteration zone has an average diameter of $1.5 \mathrm{~m}$, at least in the interval of exposure during the present last stage of digging, and show gradational contacts with the unaltered pegmatite. In this alteration zone, the majority of the elbaite tourmaline of different colors occurs, most of them of good gem quality, and always found associated with cleavelandite, lepidolite, bipyramidal light-smoked quartz crystals, which are in some cases cut by green tourmaline, iridescent manganotantalite, and dark green to green microlite, which usually occurs associate with cleavelandite, are also present in this replacement body. Some microlite crystals are translucent, usually showing flat habit, but crystals with typical octahedral forms are also present.

This replacement body clearly represents an enrichment phase of the Alto Quixaba pegmatite, as indicated by the presence of $\mathrm{Na}, \mathrm{Li}$, and other elements such as B, $\mathrm{Zn}, \mathrm{Mg}, \mathrm{Mn}, \mathrm{Cu}$, Ti e F, which are incorporated in the elbaite tourmaline, besides 


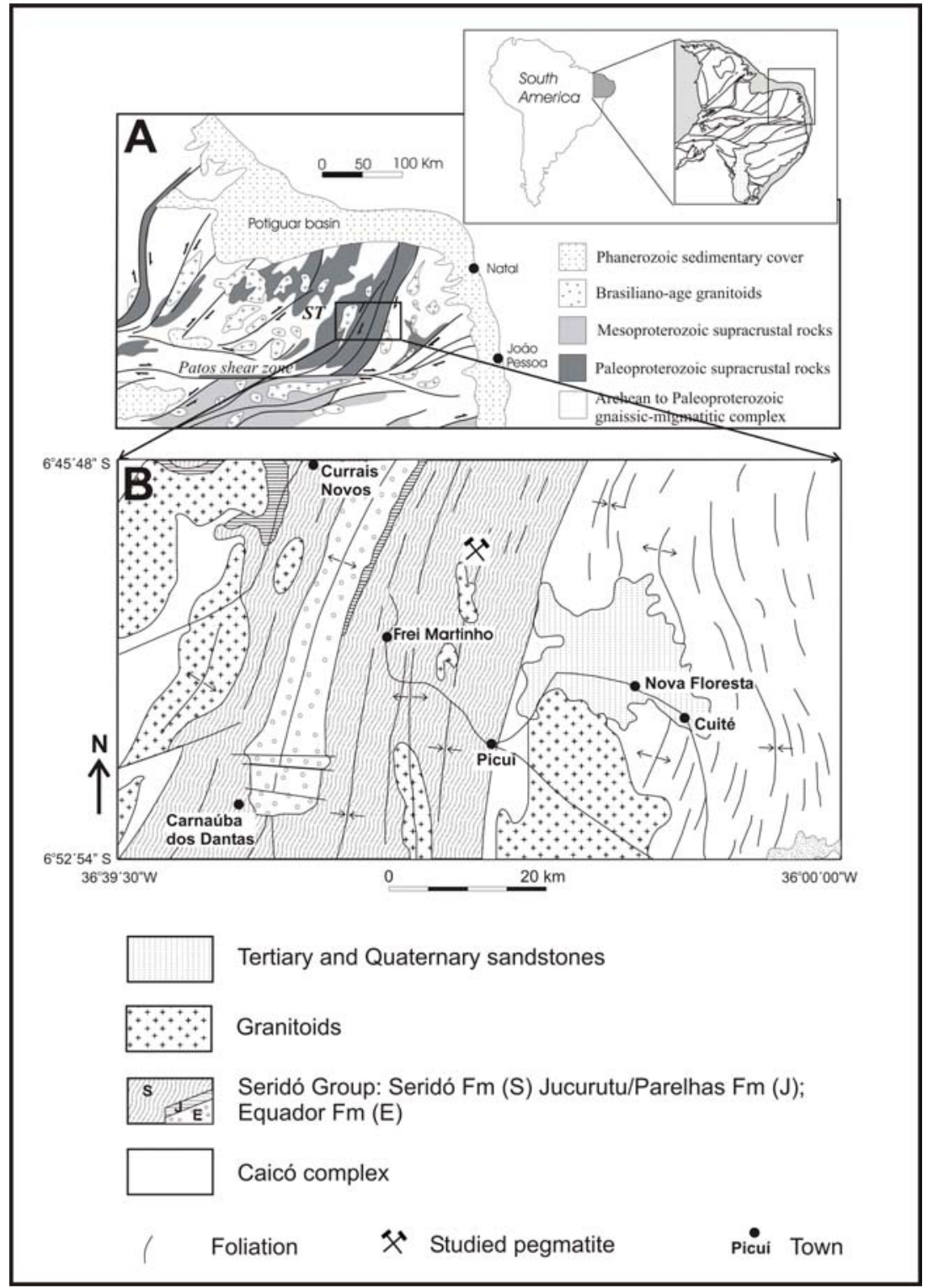

Fig. 1 - Location of the Borborema province in northeastern Brazil (insert of A); (A) geological sketch of the Seridó foldbelt; (B) location of the Alto Quixaba pegmatite, intruded into biotite schists of the Seridó Formation of the Seridó Group. Geological units shown in (B) are from E.F. Jardim de Sá (unpublished data).

some metallic elements in tantalates (A.C.M. Ferreira, unpublished data). This replacement body is texturally and chemically similar to alteration zones described in granitic pegmatitic bodies elsewhere, which are interpreted as representing the final resid- ual water-rich melt rich in silica, alumina, water, halogens, alkali and lithophile elements, and reflect structural and chemical activities during pegmatite crystallization (e.g. Guilbert and Park 1986). The presence of alteration zone coupled with rare miner- 


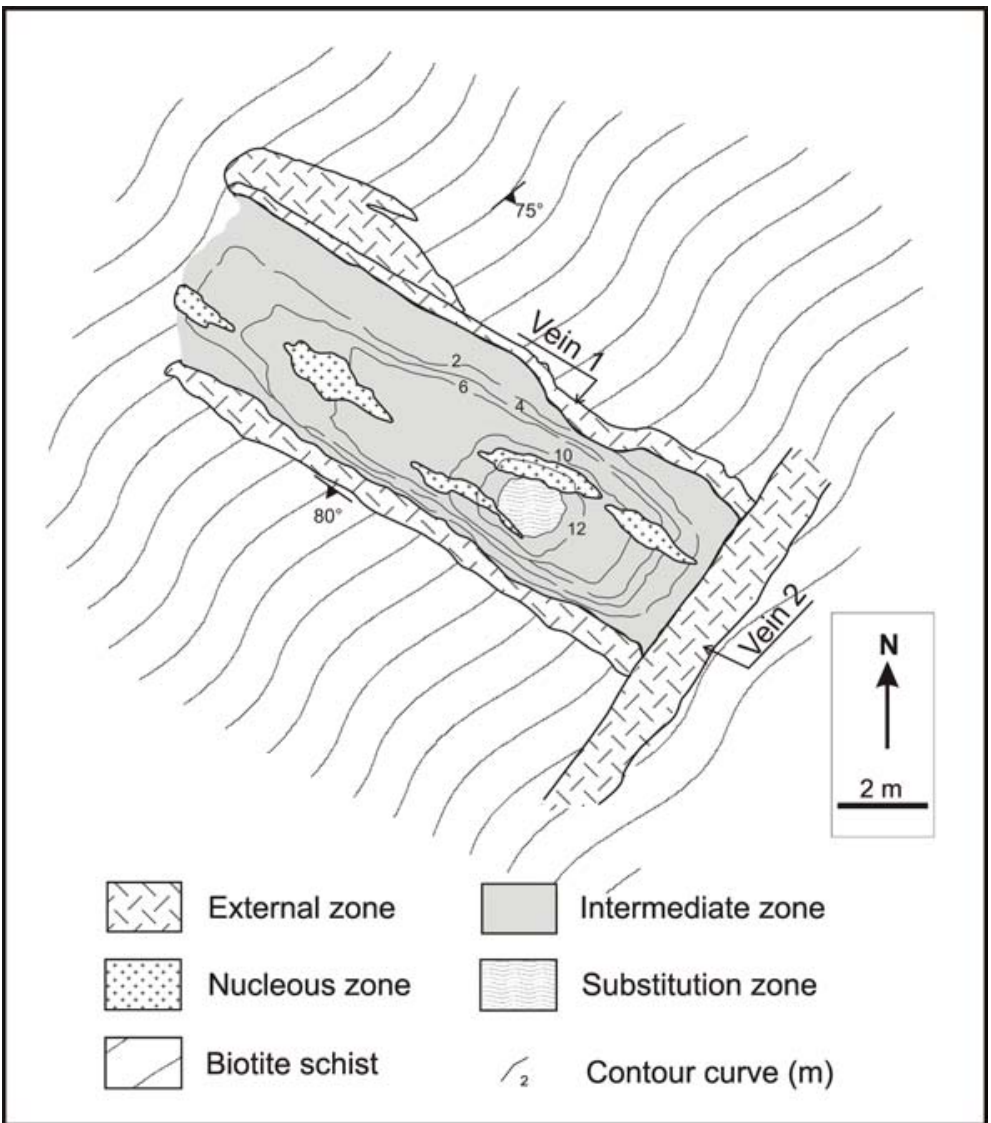

Fig. 2 - Geological sketch of the Alto Quixaba pegmatite showing its textural and mineralogical zonation (A.C.M. Ferreira, unpublished data). Descriptions of each zone are provided in the text.

als such as elbaite, manganotantalite and microlite, among others, define this as a zoned, complex pegmatitic body, according to of Cameron et al. (1949) and Norton (1983) criteria.

\section{SAMPLES AND ANALYTICAL METHODS}

Over 80 tourmaline samples, crystals and fragments of a single crystal, many of them of gem quality, have been collected from the Alto Quixaba pegmatite. The size of individual tourmaline crystals varies from few $\mathrm{cm}$ up to $6 \mathrm{~cm}$ in length. Twenty six gem-quality crystals varying in weigh from 0.35 to 5.4 carats were cut and polished for determination of pleochroism, refractive index and birefringence, at the Gemological Center of Northeast, Federal
University of Campina Grande. Refractive indexes have been measured in an Eickhorst refractometer for solids model GEMled. For each sample, 10-15 measurements have been made.

The unit-cell study of selected samples of four different colors was done using powder diagrams obtained in a diffractometer Rigaku Denki, at the Department of Mining Engineering, Federal University of Pernambuco. Radiation used was $\mathrm{Cu}$ $\mathrm{K} \alpha$, a graphite monochromator filter, in the following conditions of operation: $30 \mathrm{~m} \AA$ and $40 \mathrm{kV}$, speed of the goniometer of $4 \%$ minute, and paper of $40 \mathrm{~mm} /$ minute, with the diffractometer varying from approximately $10^{\circ}$ to $70^{\circ}$.

The cell parameters, calculated using the 
method of Ito (Vilarroel-Leo 1971), are displayed in Table I, which also shows representative powder diagrams for blue and green elbaites of the Alto Quixaba pegmatite, as well as the values for an elbaite of the JCPDS (Joint Committee of Powder Diffraction Standard) system, number 21251, for comparison.

Electron microprobe and ICP-AES chemical analyses have been performed in five samples of elbaites of different colors. Chemical analyzes were performed at the University Pierre et Marie Curie, France, using an electronic microprobe CAMECA model SX-50, in the following conditions for all analyzed elements, except for B: $15 \mathrm{kV}, 10 \mathrm{nA}, 10 \mu \mathrm{m}$ diameter of the electron bean, and $20 \mathrm{~s}$ counting time. A $10 \mathrm{kV}$ voltage and a counting time of $5 \mathrm{~s}$ were used for the analyses of B. Reference materials used were: diopside ( $\mathrm{Si}, \mathrm{Mg}, \mathrm{Ca}$ ), synthetic hematite $(\mathrm{Fe})$, orthoclase $(\mathrm{K}, \mathrm{Al})$, pyrophanite $(\mathrm{Ti})$, albite (Na) and boron nitrate (B). Minor elements have been corrected using conventional programs PAP e ZAF. Lithium and other minor elements have been analyzed by ICP-AES at the Saint-Etienne School of Mines, France. Structural formulae were calculated on the basis of 29 oxygens, assuming a complete occupation of 4 anions in the hydroxila site, following the procedures described by Henry and Guidotti (1985). Results are displayed in Tables II and III.

\section{PHYSICAL AND OPTICAL CHARACTERISTICS}

The elbaites from the Alto Quixaba pegmatite occur as elongate prismatic crystals, with rounded faces, and typical striations parallel to the c-axis; some crystals are up to $150 \mathrm{~g}$ in weigh. They are mainly blue, violet-blue, greenish blue, green and dark green crystals; some of them are translucent when observed perpendicular through the optical c axis. Two major types of zoning are present: (a) perpendicular to the c-axis, with each extremity showing one color (e.g. blue and green), and (b) along the c-axis, giving rise to concentric zoning, in which the core is greenish blue while the rim is green. In this case, tricolor zoning can also be observed, usually with colors ranging from violet through blue and green, or deep pink core surrounded by a green or blue zone, and a thin pink rim.

They exhibit vitreous luster, no cleavage, and hardness around 7 in the Mohr scale of hardness. Density, determined by the hydrostatic method, varies from 3.05 to 3.10 , with an average value of $3.07 \pm 0.01$, within the range for elbaites studied by Donnay and Barton (1972), who reported densities between 2.84 and 3.10. The studied elbaites have strong pleochroism and uniaxial negative optical character. Measured refractive indexes are $\eta_{\epsilon}=1.619-1.622$ and $\eta_{\omega}=1.639-1.643$, and birefringence $(\Delta)$ is between 0.019 and 0.021 . These values are in the same range for the elbaites described by Dietrich (1985), who reported values of $\epsilon \sim 1.622, \eta \sim 1.641$ and $\Delta \sim 0.019$. Unit-cell dimensions $(a \mathrm{o}=15.845 \AA$; $c \mathrm{o}=7.085 \AA$; V $=$ $1540.476 \AA$ ) of the studied tourmalines correspond closely to the elbaite reference parameters.

The Kerez effect of cut tourmalines was reported for the first time by Mitchell (1967, in Dietrich 1985) and later studied in detail by Schiffmann (1972, 1975, in Dietrich 1985). Tourmalines that present Kerez effect exhibit two, three or four refractive index bands under a refractrometer. Among the 26 cut tourmalines examined here, the Kerez effect was observed in two dark green crystals that showed two refractive index bands. This is consistent with the observation by Anderson (1980) and Read (1982) that related the Kerez effect to green tourmalines. According to Dietrich (1985), this phenomenon seems to be provoked by overheating of crystal faces during cutting, and the correct refractive index is the largest measured value.

Several cut samples of tourmalines observed under a gemological microscope showed to be free of inclusions. Most samples display only inclusions typical of tourmalines such as channels or fine tubes, known as trinchites (Gübelin 1979); finger prints, which are unoriented thin capillary tubes, usually in nets filled with liquids; biphase inclusions, which are according to Gübelin (1979) twophase trinchites, and strain or mechanical fractures (A.C.M. Ferreira, unpublished data). 
TABLE I

Powder diagram of gem-quality tourmalines from the Alto Quixaba pegmatite. Data for elbaite of the JCPDS is shown for comparison.

\begin{tabular}{|c|c|c|c|c|c|c|c|c|c|c|}
\hline \multirow[t]{2}{*}{ hkl } & \multicolumn{2}{|c|}{ Green } & \multicolumn{2}{|c|}{ Blue } & \multicolumn{2}{|c|}{ Violet blue } & \multicolumn{2}{|c|}{ Dark green } & \multicolumn{2}{|c|}{$\begin{array}{c}\text { Elbaite } \\
\text { (JCPDS- } \\
(21251\end{array}$} \\
\hline & $\mathrm{d}(\AA)$ & $\mathrm{I} / \mathrm{I}_{0}$ & $\mathrm{~d}(\AA)$ & $\mathrm{I} / \mathrm{I}_{0}$ & $\mathrm{~d}(\AA)$ & $\mathrm{I} / \mathrm{I}_{0}$ & $\mathrm{~d}(\AA)$ & $\mathrm{I} / \mathrm{I}_{0}$ & $\mathrm{~d}(\AA)$ & $\mathrm{I} / \mathrm{I}_{0}$ \\
\hline 101 & 6.28 & 15 & 6.30 & 20 & 6.28 & 20 & 6.26 & 15 & 6.31 & 20 \\
\hline 111 & - & - & - & - & - & - & - & - & 5.29 & 20 \\
\hline 021 & 4.93 & 25 & 4.93 & 15 & 4.93 & 35 & 4.93 & 15 & - & - \\
\hline 300 & 4.56 & 10 & 4.55 & 10 & 4.56 & 5 & 4.56 & 5 & - & - \\
\hline 211 & 4.19 & 25 & 4.18 & 30 & 4.19 & 30 & 4.17 & 20 & 4.19 & 25 \\
\hline 220 & 3.95 & 45 & 3.95 & 45 & 3.95 & 35 & 3.95 & 35 & 3.96 & 60 \\
\hline 400 & 3.43 & 100 & 3.43 & 60 & 3.44 & 100 & 3.43 & 55 & 3.44 & 50 \\
\hline 131 & 3.35 & 10 & 3.35 & 15 & 3.35 & 10 & 3.34 & 10 & 3.35 & 16 \\
\hline 410 & 2.99 & 10 & 2.99 & 20 & 2.99 & 15 & 2.98 & 10 & 2.994 & 35 \\
\hline 122 & 2.94 & 60 & 2.93 & 75 & 2.93 & 55 & 2.92 & 100 & 2.930 & 40 \\
\hline 051 & 2.56 & 55 & 2.56 & 100 & 2.56 & 50 & 2.55 & 30 & 2.560 & 100 \\
\hline 003 & 2.37 & 15 & 2.37 & 30 & 2.37 & 5 & 2.36 & 15 & - & - \\
\hline 103 & 2.33 & 10 & 2.33 & 15 & 2.33 & 15 & 2.32 & 15 & 2.333 & 20 \\
\hline 431 & 2.15 & 10 & 2.15 & 15 & 2.15 & 10 & 2.15 & 10 & 2.150 & 16 \\
\hline 521 & 2.103 & 15 & 2.106 & 20 & 2.103 & 15 & 2.094 & 15 & 2.099 & 20 \\
\hline 152 & 2.027 & 20 & 2.030 & 75 & 2.027 & 30 & 2.027 & 25 & 2.025 & 20 \\
\hline 530 & & & & & & & & & & \\
\hline 700 & 1.981 & $<5$ & 1.981 & $<5$ & 1.977 & 5 & 1.977 & 10 & 1.960 & 25 \\
\hline 342 & 1.910 & 55 & 1.910 & 30 & 1.906 & 20 & 1.906 & 30 & - & - \\
\hline 522 & 1.862 & 5 & 1.859 & 10 & 1.859 & 5 & 1.859 & 10 & 1.868 & 25 \\
\hline 004 & 1.767 & 20 & 1.767 & 10 & 1.770 & 10 & 1.770 & 5 & 1.776 & 45 \\
\hline 550 & 1.588 & 5 & 1.587 & 10 & 1.587 & 5 & 1.587 & 20 & - & - \\
\hline 452 & 1.577 & 5 & 1.580 & 10 & 1.587 & 5 & 1.587 & 20 & 1.575 & 25 \\
\hline 641 & 1.539 & 5 & 1.539 & $<5$ & 1.540 & $<5$ & - & - & 1.537 & 20 \\
\hline
\end{tabular}

\section{CRYSTAL CHEMISTRY AND STRUCTURE}

Tourmaline can be defined as a chemical and structurally complex borosilicate, with a composition, although quite variable, represented by the general formula $\mathrm{XY}_{3} \mathrm{Z}_{6}\left(\mathrm{BO}_{3}\right)_{3}\left(\mathrm{~T}_{6} \mathrm{O}_{18}\right) \mathrm{W}_{4}$ (Hawthorne 1996), the crystallographic sites being partially or totally occupied. The crystal structure of tourmaline is such that it allows substitution of a variety of ions with respect to both size and charge (Jolliff et al. 1986) in a single site or as coupled substitutions over several sites. The $\mathrm{X}$-structural site is occupied by alkali cations, usually $\mathrm{Na}^{+}, \mathrm{Ca}^{2+}, \mathrm{K}^{+}$, and $\mathrm{Mg}^{2+}$, or even presenting total or partial vacancies. The $\mathrm{Y}$ site permits diverse and extensive substitutions, involving monovalent, divalent, trivalent and quadrivalent cations, such as $\mathrm{Fe}^{2+}, \mathrm{Mg}^{2+},\left(\mathrm{Al}^{3+}+\right.$ $\left.\mathrm{Li}^{+}\right), \mathrm{Fe}^{3+}, \mathrm{Mn}^{2+}, \mathrm{Ca}^{2+}, \mathrm{Cr}^{3+}, \mathrm{Ti}^{4+}$. The $\mathrm{Z}$ site is usually occupied by $\mathrm{Al}^{3+}$. In elbaite, the $\mathrm{Y}$ site is predominantly occupied with $\mathrm{Al}$ and $\mathrm{Li}$, but may contain $\mathrm{Fe}^{3+}, \mathrm{Cr}^{3+}, \mathrm{V}^{3+}$ or $\mathrm{Mg}$, in dravite predomi- 
TABLE II

Electron microprobe analyses of blue and green elbaites from the Alto Quixaba pegmatite, northeastern Brazil. Structural formulae calculated on the basis of 29 oxygens, assuming a complete occupation of 4 anions in the hydroxila site. $\mathrm{Li}_{2} \mathrm{O}$ contents analyzed by ICP-AES.

\begin{tabular}{|c|c|c|c|c|c|c|}
\hline \multirow{2}{*}{$\begin{array}{c}\text { Blue } \\
\text { elbaite }\end{array}$} & \multicolumn{6}{|c|}{ Crystal 1} \\
\hline & 1 & 2 & 3 & 4 & 5 & 6 \\
\hline $\mathrm{SiO}_{2}$ & 39.08 & 37.76 & 38.25 & 37.62 & 37.41 & 37.38 \\
\hline $\mathrm{TiO}_{2}$ & 0.04 & 0.04 & 0.07 & 0.02 & 0.06 & 0.07 \\
\hline $\mathrm{Al}_{2} \mathrm{O}_{3}$ & 38.25 & 38.49 & 38.16 & 38.18 & 37.67 & 37.72 \\
\hline $\mathrm{FeO}$ & 2.85 & 2.85 & 2.90 & 2.95 & 3.16 & 3.13 \\
\hline $\mathrm{MnO}$ & 2.86 & 2.80 & 2.88 & 2.70 & 2.85 & 3.12 \\
\hline $\mathrm{CaO}$ & 0.25 & 0.33 & 0.31 & 0.29 & 0.27 & 0.27 \\
\hline $\mathrm{MgO}$ & 0.00 & 0.01 & 0.00 & 0.02 & 0.01 & 0.00 \\
\hline $\mathrm{Na}_{2} \mathrm{O}$ & 2.82 & 2.56 & 2.55 & 2.65 & 2.59 & 2.65 \\
\hline $\mathrm{K}_{2} \mathrm{O}$ & 0.00 & 0.00 & 0.00 & 0.00 & 0.05 & 0.00 \\
\hline $\mathrm{CuO}$ & 0.01 & 0.01 & 0.00 & 0.00 & 0.04 & 0.04 \\
\hline $\mathrm{ZnO}$ & 0.41 & 0.50 & 0.43 & 0.45 & 0.47 & 0.47 \\
\hline $\mathrm{B}_{2} \mathrm{O}_{3}$ & 11.31 & 10.93 & 11.07 & 10.89 & 10.83 & 10.82 \\
\hline $\mathrm{Li}_{2} \mathrm{O}$ & 1.74 & 1.74 & 1.74 & 1.74 & 1.74 & 1.74 \\
\hline Total & 99.62 & 98.02 & 98.36 & 97.51 & 97.15 & 97.41 \\
\hline $\mathrm{Si}$ & 6.07 & 5.97 & 6.02 & 5.98 & 5.98 & 5.97 \\
\hline $\mathrm{Ti}$ & 0.00 & 0.00 & 0.01 & 0.00 & 0.01 & 0.01 \\
\hline $\mathrm{Al}$ & 7.00 & 7.17 & 7.08 & 7.15 & 7.10 & 7.10 \\
\hline $\mathrm{Fe}$ & 0.37 & 0.38 & 0.38 & 0.39 & 0.42 & 0.42 \\
\hline $\mathrm{Mn}$ & 0.38 & 0.38 & 0.38 & 0.36 & 0.39 & 0.42 \\
\hline $\mathrm{Ca}$ & 0.04 & 0.06 & 0.05 & 0.05 & 0.05 & 0.05 \\
\hline $\mathrm{Mg}$ & 0.00 & 0.00 & 0.00 & 0.00 & 0.00 & 0.00 \\
\hline $\mathrm{Na}$ & 0.85 & 0.78 & 0.78 & 0.82 & 0.80 & 0.82 \\
\hline $\mathrm{K}$ & 0.00 & 0.00 & 0.00 & 0.00 & 0.01 & 0.00 \\
\hline $\mathrm{Cu}$ & 0.00 & 0.00 & 0.00 & 0.00 & 0.00 & 0.00 \\
\hline $\mathrm{Zn}$ & 0.05 & 0.06 & 0.05 & 0.05 & 0.06 & 0.06 \\
\hline $\mathrm{B}$ & 3.03 & 2.98 & 3.01 & 2.99 & 2.99 & 2.98 \\
\hline $\mathrm{Li}$ & 1.08 & 1.10 & 1.10 & 1.11 & 1.12 & 1.12 \\
\hline Total & 17.79 & 17.79 & 17.77 & 17.80 & 17.81 & 17.83 \\
\hline $\mathrm{Na}$ & 0.85 & 0.78 & 0.78 & 0.82 & 0.80 & 0.82 \\
\hline $\mathrm{Ca}$ & 0.04 & 0.06 & 0.05 & 0.05 & 0.05 & 0.05 \\
\hline $\mathrm{K}$ & 0.00 & 0.00 & 0.00 & 0.00 & 0.01 & 0.00 \\
\hline Vac. & 0.11 & 0.16 & 0.17 & 0.13 & 0.14 & 0.13 \\
\hline Total X & 1.00 & 1.00 & 1.00 & 1.00 & 1.00 & 1.00 \\
\hline $\mathrm{Li}$ & 1.08 & 1.10 & 1.10 & 1.11 & 1.12 & 1.12 \\
\hline $\mathrm{Al}(\mathrm{Y})$ & 1.00 & 1.14 & 1.08 & 1.13 & 1.08 & 1.07 \\
\hline $\mathrm{Fe}$ & 0.37 & 0.38 & 0.38 & 0.39 & 0.42 & 0.42 \\
\hline $\mathrm{Mn}$ & 0.38 & 0.38 & 0.38 & 0.36 & 0.39 & 0.42 \\
\hline $\mathrm{Mg}$ & 0.00 & 0.00 & 0.00 & 0.00 & 0.00 & 0.00 \\
\hline $\mathrm{Cu}$ & 0.00 & 0.00 & 0.00 & 0.00 & 0.00 & 0.00 \\
\hline $\mathrm{Zn}$ & 0.05 & 0.06 & 0.05 & 0.05 & 0.06 & 0.06 \\
\hline $\mathrm{Ti}$ & 0.00 & 0.00 & 0.01 & 0.00 & 0.01 & 0.01 \\
\hline Total Y & 2.88 & 3.06 & 3.00 & 3.06 & 3.08 & 3.09 \\
\hline $\mathrm{Al}(\mathrm{Z})$ & 6.00 & 6.00 & 6.00 & 6.00 & 6.00 & 6.00 \\
\hline Total Z & 6.00 & 6.00 & 6.00 & 6.00 & 6.00 & 6.00 \\
\hline $\mathrm{Si}$ & 6.07 & 5.97 & 6.02 & 5.98 & 5.98 & 5.97 \\
\hline $\mathrm{Al}(\mathrm{T})$ & & 0.03 & & 0.02 & 0.02 & 0.03 \\
\hline \multicolumn{7}{|l|}{ Total T } \\
\hline $\mathrm{B}$ & 3.03 & 2.98 & 3.01 & 2.99 & 2.99 & 2.98 \\
\hline Total B OH+F=4 & 3.03 & 2.98 & 3.01 & 2.99 & 2.99 & 2.98 \\
\hline
\end{tabular}


TABLE II (continuation)

\begin{tabular}{|c|c|c|c|c|c|c|c|c|c|c|}
\hline \multirow{2}{*}{$\begin{array}{c}\text { Blue } \\
\text { elbaite }\end{array}$} & \multicolumn{5}{|c|}{ Crystal 2} & \multicolumn{5}{|c|}{ Crystal 3} \\
\hline & 1 & 2 & 3 & 4 & 5 & 1 & 2 & 3 & 4 & 5 \\
\hline $\mathrm{SiO}_{2}$ & 36.87 & 37.20 & 37.78 & 37.60 & 37.14 & 37.62 & 37.35 & 36.74 & 38.40 & 37.47 \\
\hline $\mathrm{TiO}_{2}$ & 0.05 & 0.06 & 0.04 & 0.05 & 0.04 & 0.00 & 0.00 & 0.00 & 0.02 & 0.01 \\
\hline $\mathrm{Al}_{2} \mathrm{O}_{3}$ & 38.65 & 37.58 & 38.69 & 38.84 & 37.95 & 37.89 & 38.45 & 38.44 & 38.48 & 37.00 \\
\hline $\mathrm{FeO}$ & 3.93 & 3.76 & 3.91 & 3.96 & 3.80 & 2.45 & 2.51 & 2.70 & 2.62 & 2.63 \\
\hline $\mathrm{MnO}$ & 2.53 & 2.34 & 2.44 & 2.36 & 2.56 & 2.26 & 2.08 & 1.93 & 2.10 & 2.08 \\
\hline $\mathrm{CaO}$ & 0.25 & 0.30 & 0.24 & 0.21 & 0.20 & 0.15 & 0.19 & 0.19 & 0.21 & 0.12 \\
\hline $\mathrm{MgO}$ & 0.02 & 0.03 & 0.02 & 0.01 & 0.02 & 0.00 & 0.01 & 0.01 & 0.00 & 0.00 \\
\hline $\mathrm{Na}_{2} \mathrm{O}$ & 2.48 & 2.55 & 2.66 & 2.64 & 2.55 & 2.79 & 2.63 & 2.62 & 2.73 & 2.83 \\
\hline $\mathrm{K}_{2} \mathrm{O}$ & 0.00 & 0.01 & 0.02 & 0.00 & 0.01 & 0.02 & 0.01 & 0.01 & 0.02 & 0.01 \\
\hline $\mathrm{CuO}$ & 0.00 & 0.02 & 0.00 & 0.00 & 0.00 & 0.02 & 0.00 & 0.00 & 0.02 & 0.00 \\
\hline $\mathrm{ZnO}$ & 0.39 & 0.43 & 0.43 & 0.36 & 0.48 & 2.82 & 2.73 & 2.95 & 2.90 & 2.95 \\
\hline $\mathrm{B}_{2} \mathrm{O}_{3}$ & 10.67 & 10.77 & 10.94 & 10.88 & 10.75 & 10.89 & 10.81 & 10.63 & 11.12 & 10.85 \\
\hline $\mathrm{Li}_{2} \mathrm{O}$ & 1.81 & 1.81 & 1.81 & 1.81 & 1.81 & 1.59 & 1.59 & 1.59 & 1.59 & 1.59 \\
\hline Total & 97.65 & 96.86 & 98.98 & 98.72 & 97.31 & 98.50 & 98.36 & 97.81 & 100.21 & 97.54 \\
\hline $\mathrm{Si}$ & 5.88 & 5.97 & 5.94 & 5.92 & 5.94 & 5.98 & 5.93 & 5.89 & 5.99 & 6.01 \\
\hline $\mathrm{Ti}$ & 0.01 & 0.01 & 0.00 & 0.01 & 0.00 & 0.00 & 0.00 & 0.00 & 0.00 & 0.00 \\
\hline $\mathrm{Al}$ & 7.27 & 7.11 & 7.17 & 7.21 & 7.15 & 7.10 & 7.20 & 7.26 & 7.08 & 7.00 \\
\hline $\mathrm{Fe}$ & 0.52 & 0.50 & 0.51 & 0.52 & 0.51 & 0.33 & 0.33 & 0.36 & 0.34 & 0.35 \\
\hline $\mathrm{Mn}$ & 0.34 & 0.32 & 0.32 & 0.31 & 0.35 & 0.30 & 0.28 & 0.26 & 0.28 & 0.28 \\
\hline $\mathrm{Ca}$ & 0.04 & 0.05 & 0.04 & 0.04 & 0.03 & 0.03 & 0.03 & 0.03 & 0.04 & 0.02 \\
\hline $\mathrm{Mg}$ & 0.00 & 0.01 & 0.00 & 0.00 & 0.00 & 0.00 & 0.00 & 0.00 & 0.00 & 0.00 \\
\hline $\mathrm{Na}$ & 0.77 & 0.79 & 0.81 & 0.81 & 0.79 & 0.86 & 0.81 & 0.81 & 0.83 & 0.88 \\
\hline K & 0.00 & 0.00 & 0.00 & 0.00 & 0.00 & 0.00 & 0.00 & 0.00 & 0.00 & 0.00 \\
\hline $\mathrm{Cu}$ & 0.00 & 0.00 & 0.00 & 0.00 & 0.00 & 0.00 & 0.00 & 0.00 & 0.00 & 0.00 \\
\hline $\mathrm{Zn}$ & 0.05 & 0.05 & 0.05 & 0.04 & 0.06 & 0.33 & 0.32 & 0.35 & 0.33 & 0.35 \\
\hline B & 2.94 & 2.98 & 2.97 & 2.96 & 2.97 & 2.99 & 2.96 & 2.94 & 2.99 & 3.01 \\
\hline $\mathrm{Li}$ & 1.16 & 1.17 & 1.14 & 1.15 & 1.16 & 1.01 & 1.01 & 1.02 & 1.00 & 1.02 \\
\hline Total & 17.82 & 17.79 & 17.83 & 17.82 & 17.81 & 17.91 & 17.88 & 17.91 & 17.89 & 17.91 \\
\hline $\mathrm{Na}$ & 0.77 & 0.79 & 0.81 & 0.81 & 0.79 & 0.86 & 0.81 & 0.81 & 0.83 & 0.88 \\
\hline $\mathrm{Ca}$ & 0.04 & 0.05 & 0.04 & 0.04 & 0.03 & 0.03 & 0.03 & 0.03 & 0.04 & 0.02 \\
\hline K & 0.00 & 0.00 & 0.00 & 0.00 & 0.00 & 0.00 & 0.00 & 0.00 & 0.00 & 0.00 \\
\hline Vac. & 0.19 & 0.15 & 0.15 & 0.16 & 0.17 & 0.11 & 0.16 & 0.15 & 0.13 & 0.10 \\
\hline Total X & 1.00 & 1.00 & 1.00 & 1.00 & 1.00 & 1.00 & 1.00 & 1.00 & 1.00 & 1.00 \\
\hline $\mathrm{Li}$ & 1.16 & 1.17 & 1.14 & 1.15 & 1.16 & 1.01 & 1.01 & 1.02 & 1.00 & 1.02 \\
\hline $\mathrm{Al}(\mathrm{Y})$ & 1.15 & 1.08 & 1.11 & 1.13 & 1.09 & 1.08 & 1.13 & 1.15 & 1.07 & 1.00 \\
\hline $\mathrm{Fe}$ & 0.52 & 0.50 & 0.51 & 0.52 & 0.51 & 0.33 & 0.33 & 0.36 & 0.34 & 0.35 \\
\hline $\mathrm{Mn}$ & 0.34 & 0.32 & 0.32 & 0.31 & 0.35 & 0.30 & 0.28 & 0.26 & 0.28 & 0.28 \\
\hline $\mathrm{Mg}$ & 0.00 & 0.01 & 0.00 & 0.00 & 0.00 & 0.00 & 0.00 & 0.00 & 0.00 & 0.00 \\
\hline $\mathrm{Cu}$ & 0.00 & 0.00 & 0.00 & 0.00 & 0.00 & 0.00 & 0.00 & 0.00 & 0.00 & 0.00 \\
\hline $\mathrm{Zn}$ & 0.05 & 0.05 & 0.05 & 0.04 & 0.06 & 0.33 & 0.32 & 0.35 & 0.33 & 0.35 \\
\hline $\mathrm{Ti}$ & 0.01 & 0.01 & 0.00 & 0.01 & 0.00 & 0.00 & 0.00 & 0.00 & 0.00 & 0.00 \\
\hline Total Y & 3.23 & 3.14 & 3.15 & 3.16 & 3.17 & 3.05 & 3.08 & 3.14 & 3.03 & 3.01 \\
\hline $\mathrm{Al}(\mathrm{Z})$ & 6.00 & 6.00 & 6.00 & 6.00 & 6.00 & 6.00 & 6.00 & 6.00 & 6.00 & 6.00 \\
\hline Total Z & 6.00 & 6.00 & 6.00 & 6.00 & 6.00 & 6.00 & 6.00 & 6.00 & 6.00 & 6.00 \\
\hline $\mathrm{Si}$ & 5.88 & 5.97 & 5.94 & 5.92 & 5.94 & 5.98 & 5.93 & 5.89 & 5.99 & 6.01 \\
\hline $\mathrm{Al}(\mathrm{T})$ & 0.12 & 0.03 & 0.06 & 0.08 & 0.06 & 0.02 & 0.07 & 0.11 & 0.01 & \\
\hline Total T & 6.00 & 6.00 & 6.00 & 6.00 & 6.00 & 6.00 & 6.00 & 6.00 & 6.00 & 6.01 \\
\hline B & 2.94 & 2.98 & 2.97 & 2.96 & 2.97 & 2.99 & 2.96 & 2.94 & 2.99 & 3.01 \\
\hline Total $\mathrm{B} \mathrm{OH}+\mathrm{F}=4$ & 2.942 .98 & 2.97 & 2.96 & 2.97 & 2.99 & 2.96 & 2.94 & 2.99 & 3.01 & \\
\hline
\end{tabular}

nates $\mathrm{Mg}$, and in schorl, $\mathrm{Fe}^{2+}$. The $\mathrm{B}$ site, occupied by $\mathrm{B}$, is of regular triangular coordination, and apparently does not suffer any substitution. The $\mathrm{T}$ site is usually filled by $\mathrm{Si}$, but can also accommodate $\mathrm{Al}$. In the $\mathrm{W}$ site $\mathrm{OH}-$ can be substituted for $\mathrm{F}^{-}$or $\mathrm{O}^{2-}$
(Foit and Rosenberg 1979, Povondra 1981, Werding and Schreyer 1984, Gallagher 1988, Burt 1989, MacDonald and Hawthorne 1995).

Most natural tourmalines belong to two solid solutions series: schorl-dravite and schorl-elbaite, 
TABLE II (continuation)

\begin{tabular}{|c|c|c|c|c|c|c|c|c|}
\hline \multirow{2}{*}{$\begin{array}{c}\text { Green } \\
\text { e l b a ite }\end{array}$} & \multicolumn{4}{|c|}{ Crystal 1} & \multicolumn{4}{|c|}{ Crystal 2} \\
\hline & 1 & 2 & 3 & 4 & 1 & 2 & 3 & 4 \\
\hline $\mathrm{SiO}_{2}$ & 37.03 & 37.26 & 37.37 & 36.63 & 37.70 & 37.79 & 37.52 & 37.51 \\
\hline $\mathrm{TiO}_{2}$ & 0.11 & 0.15 & 0.13 & 0.10 & 0.07 & 0.11 & 0.07 & 0.06 \\
\hline $\mathrm{Al}_{2} \mathrm{O}_{3}$ & 38.15 & 38.79 & 38.87 & 38.98 & 37.98 & 38.09 & 38.46 & 38.68 \\
\hline $\mathrm{FeO}$ & 5.10 & 5.26 & 4.65 & 5.14 & 2.90 & 2.81 & 2.85 & 2.73 \\
\hline $\mathrm{MnO}$ & 1.74 & 1.75 & 1.63 & 1.80 & 1.80 & 0.96 & 1.99 & 2.02 \\
\hline $\mathrm{CaO}$ & 0.12 & 0.14 & 0.16 & 0.10 & 0.18 & 0.15 & 0.17 & 0.15 \\
\hline $\mathrm{MgO}$ & 0.14 & 0.12 & 0.11 & 0.14 & 0.00 & 0.00 & 0.01 & 0.00 \\
\hline $\mathrm{Na}_{2} \mathrm{O}$ & 2.66 & 2.56 & 2.76 & 2.58 & 2.78 & 2.72 & 2.90 & 2.73 \\
\hline $\mathrm{K}_{2} \mathrm{O}$ & 0.03 & 0.04 & 0.02 & 0.03 & 0.00 & 0.00 & 0.00 & 0.00 \\
\hline $\mathrm{CuO}$ & 0.00 & 0.03 & 0.00 & 0.00 & 0.00 & 0.00 & 0.00 & 0.00 \\
\hline $\mathrm{ZnO}$ & 0.25 & 0.20 & 0.20 & 0.27 & 2.96 & 3.01 & 2.96 & 2.99 \\
\hline $\mathrm{B}_{2} \mathrm{O}_{3}$ & 10.72 & 10.79 & 10.82 & 10.60 & 10.91 & 10.94 & 10.86 & 10.28 \\
\hline $\mathrm{Li}_{2} \mathrm{O}$ & 1.67 & 1.67 & 1.67 & 1.67 & 1.67 & 1.67 & 1.67 & 1.67 \\
\hline Total & 97.72 & 98.76 & 98.39 & 98.04 & 98.95 & 98.25 & 99.46 & 98.82 \\
\hline $\mathrm{Si}$ & 5.91 & 5.89 & 5.91 & 5.84 & 5.97 & 5.99 & 5.92 & 5.96 \\
\hline $\mathrm{Ti}$ & 0.01 & 0.02 & 0.02 & 0.01 & 0.01 & 0.01 & 0.01 & 0.01 \\
\hline $\mathrm{Al}$ & 7.18 & 7.23 & 7.24 & 7.32 & 7.08 & 7.12 & 7.15 & 7.25 \\
\hline $\mathrm{Fe}$ & 0.68 & 0.70 & 0.61 & 0.69 & 0.38 & 0.37 & 0.38 & 0.36 \\
\hline $\mathrm{Mn}$ & 0.24 & 0.23 & 0.22 & 0.24 & 0.24 & 0.13 & 0.27 & 0.27 \\
\hline $\mathrm{Ca}$ & 0.02 & 0.02 & 0.03 & 0.02 & 0.03 & 0.03 & 0.03 & 0.03 \\
\hline $\mathrm{Mg}$ & 0.03 & 0.03 & 0.03 & 0.03 & 0.00 & 0.00 & 0.00 & 0.00 \\
\hline $\mathrm{Na}$ & 0.82 & 0.78 & 0.85 & 0.80 & 0.85 & 0.84 & 0.89 & 0.84 \\
\hline $\mathrm{K}$ & 0.01 & 0.01 & 0.00 & 0.01 & 0.00 & 0.00 & 0.00 & 0.00 \\
\hline $\mathrm{Cu}$ & 0.00 & 0.00 & 0.00 & 0.00 & 0.00 & 0.00 & 0.00 & 0.00 \\
\hline $\mathrm{Zn}$ & 0.03 & 0.02 & 0.02 & 0.03 & 0.35 & 0.35 & 0.34 & 0.35 \\
\hline $\mathrm{B}$ & 2.95 & 2.94 & 2.95 & 2.91 & 2.98 & 2.99 & 2.96 & 2.82 \\
\hline $\mathrm{Li}$ & 1.07 & 1.06 & 1.06 & 1.07 & 1.06 & 1.06 & 1.06 & 1.06 \\
\hline Total & 17.89 & 17.88 & 17.88 & 17.90 & 17.89 & 17.83 & 17.94 & 17.89 \\
\hline $\mathrm{Na}$ & 0.82 & 0.78 & 0.85 & 0.80 & 0.85 & 0.84 & 0.89 & 0.84 \\
\hline $\mathrm{Ca}$ & 0.02 & 0.02 & 0.03 & 0.02 & 0.03 & 0.03 & 0.03 & 0.03 \\
\hline $\mathrm{K}$ & 0.01 & 0.01 & 0.00 & 0.01 & 0.00 & 0.00 & 0.00 & 0.00 \\
\hline Vac. & 0.15 & 0.18 & 0.12 & 0.18 & 0.12 & 0.14 & 0.08 & 0.13 \\
\hline Total X & 1.00 & 1.00 & 1.00 & 1.00 & 1.00 & 1.00 & 1.00 & 1.00 \\
\hline $\mathrm{Li}$ & 1.07 & 1.06 & 1.06 & 1.07 & 1.06 & 1.06 & 1.06 & 1.06 \\
\hline $\mathrm{Al}(\mathrm{Y})$ & 1.09 & 1.12 & 1.15 & 1.16 & 1.05 & 1.11 & 1.07 & 1.21 \\
\hline $\mathrm{Fe}$ & 0.68 & 0.70 & 0.61 & 0.69 & 0.38 & 0.37 & 0.38 & 0.36 \\
\hline $\mathrm{Mn}$ & 0.24 & 0.23 & 0.22 & 0.24 & 0.24 & 0.13 & 0.27 & 0.27 \\
\hline $\mathrm{Mg}$ & 0.03 & 0.03 & 0.03 & 0.03 & 0.00 & 0.00 & 0.00 & 0.00 \\
\hline $\mathrm{Cu}$ & 0.00 & 0.00 & 0.00 & 0.00 & 0.00 & 0.00 & 0.00 & 0.00 \\
\hline $\mathrm{Zn}$ & 0.03 & 0.02 & 0.02 & 0.03 & 0.35 & 0.35 & 0.34 & 0.35 \\
\hline $\mathrm{Ti}$ & 0.01 & 0.02 & 0.02 & 0.01 & 0.01 & 0.01 & 0.01 & 0.01 \\
\hline Total Y & 3.15 & 3.18 & 3.10 & 3.23 & 3.08 & 3.04 & 3.12 & 3.27 \\
\hline $\mathrm{Al}(\mathrm{Z})$ & 6.00 & 6.00 & 6.00 & 6.00 & 6.00 & 6.00 & 6.00 & 6.00 \\
\hline Total Z & 6.00 & 6.00 & 6.00 & 6.00 & 6.00 & 6.00 & 6.00 & 6.00 \\
\hline $\mathrm{Si}$ & 5.91 & 5.89 & 5.91 & 5.84 & 5.97 & 5.99 & 5.92 & 5.96 \\
\hline $\mathrm{Al}(\mathrm{T})$ & 0.09 & 0.11 & 0.09 & 0.16 & 0.03 & 0.01 & 0.08 & 0.04 \\
\hline Total T & 6.00 & 6.00 & 6.00 & 6.00 & 6.00 & 6.00 & 6.00 & 6.00 \\
\hline $\mathrm{B}$ & 2.95 & 2.94 & 2.95 & 2.91 & 2.98 & 2.99 & 2.96 & 2.82 \\
\hline Total B OH+F=4 & 2.95 & 2.94 & 2.95 & 2.91 & 2.98 & 2.99 & 2.96 & 2.82 \\
\hline
\end{tabular}

with a miscibility gap between dravite and elbaite (Deer et al. 1966). Near complete solid solutions exists between schorl and elbaite (Foit and Rosenberg 1977). Tourmalines are typically described in terms of one of these two series. The compositions of the studied elbaites in terms of Al-Fe-Mg are represented in the Fig. 3 that shows different fields of compositional range of tourmalines from different rock types, as described by Henry and Guidotti (1985). The compositions are very close to the com- 
TABLE III

Minor element contents (ppm) obtained by ICP-AES of elbaites from the Alto Quixaba pegmatite, northeastern Brazil (A.C.M. Ferreira, unpublished data).

\begin{tabular}{c|c|c|c|c|c}
\hline & blue & Violet blue & Green & Dark green & Greenish blue \\
\hline $\mathrm{Cr}$ & 644 & 897 & 708 & 885 & 825 \\
\hline $\mathrm{Ga}$ & 170 & 177 & 151 & 173 & 146 \\
\hline $\mathrm{Ni}$ & 27.8 & 20.3 & 14.1 & 16.1 & 15.3 \\
\hline $\mathrm{Be}$ & 31.2 & 19.8 & 12.3 & 11.4 & 11.9 \\
\hline $\mathrm{V}$ & 16.6 & 8.2 & 4.2 & 13.6 & 4.3 \\
\hline $\mathrm{Cu}$ & 81.3 & 47.2 & 13 & 31.3 & 7.3 \\
\hline $\mathrm{Li}$ & 8069 & 8407 & 7369 & 7736 & 7734 \\
\hline
\end{tabular}

position of ideal elbaite, falling in the field 1 of the diagram, which is ascribed to tourmalines from $\mathrm{Li}$ rich granitoid pegmatites and aplites.

The cation distribution among the crystallographic sites of the studied tourmalines, calculated on the basis of 29 oxygens indicates that the structural formula can be generically written as:

$\left(\mathrm{Na}_{0.79-0.85} \mathrm{Ca}_{0.022-0.05} \mathrm{~K}_{0.00-0.003}\right)_{\Sigma 0.83-0.88}$ $\left(\mathrm{Li}_{1.01-1.15} \mathrm{Al}_{1.01-1.06} \mathrm{Fe}_{0.37-0.66} \mathrm{Mn}_{0.22-0.38} \mathrm{Zn}_{0.026-0.34}\right.$ $\left.\mathrm{Mg}_{0.00-0.06} \mathrm{Cu}_{0.00-0.0024} \mathrm{Ti}_{0.00114-0.014}\right)_{\Sigma 2.98-3.10}$ $\mathrm{Al}_{6.00} \mathrm{~B}_{2.93-2.99}\left(\mathrm{Si}_{5.86-5.98} \mathrm{Al}_{0.02-0.14}\right)_{\Sigma 6.00} \mathrm{O}_{27}(\mathrm{OH})_{4}$ (Soares et al. 2000).

Both blue and green tourmalines present high occupancy in the $\mathrm{X}$ site, compared with the ideal value of 1.00 in elbaite, vacancies ranging from 0.08 to 0.19 apfu. All studied tourmalines are enriched in $\mathrm{Al}$ and Li. The occupancy in the Y-site is high, most samples showing excess of cations ( $\Sigma Y>3.00 \mathrm{apfu}$ ), only one sample showing vacancy (total $Y=2.88$ ). The excess of electric charge generated by the presence of $\mathrm{Al}$ in the $\mathrm{Y}$ site is equilibrated with vacancy in the $\mathrm{X}$ site, as indicated by slightly positive trend between $\mathrm{Al}(\mathrm{Y})$ and vacancy in the $\mathrm{X}$ site (Fig. 4a). The presence of $\mathrm{Al}$ in the $\mathrm{Y}$ site is not due to the substitution for Fe and Mg (Fig. 4b).

However, two coupled substitutions, alkalidefect and proton-loss substitutions, can occur to compensate the charge imbalance due to presence of $\mathrm{Al}$ in the $\mathrm{Y}$ site, which are more significant than vacancies in the X site (Foit and Rosenberg 1977). Most natural tourmalines may contain significant amounts of these two components that can be represented in a $\left(\mathrm{R}^{+}+\mathrm{R}^{2+}\right)$ vs $\mathrm{R}^{3+}$ diagram (Fig. 5a) in which $\mathrm{R}^{+}$represents the sum of alkalies

$$
\mathrm{Na}^{+}+2 \mathrm{Ca}^{2+}+\mathrm{K}^{+}, \mathrm{R}^{2+}=\mathrm{Fe}^{2+}+\mathrm{Mg}^{2+}+\mathrm{Mn}^{2+},
$$

and $\mathrm{R}^{3+}=\mathrm{Al}^{3+}+4 / 3 \mathrm{Ti}^{4+}$. The substitutions trends proposed by Gallagher (1988) are displayed in the Fig. 5a. The compositions of the studied tourmalines fall away from the loss of protons trend, to the left of the alkali-defect substitution one. Benvenuti et al. (1991) explained this behavior as due to partial occupancy of the Y site by $\mathrm{Si}$ and/or presence of additional $\mathrm{R}^{+}$cations such as Li. The tourmalines of the Alto Quixaba do not show $\mathrm{Si}$ in the $\mathrm{Y}$ site (Table II) but significant amounts of $\mathrm{Li}$ and $\mathrm{Al}$ $(\mathrm{Al}+\mathrm{Li}>2 \mathrm{apfu})$ and consequent lower $\mathrm{Mg}$ contents. Another possibility is that this diagram can not be applied for tourmalines of the schorl-elbaite series. On the other hand, this diagram shows that the compositions are rather homogeneous, which can be related to the decreasing temperature and large degree of evolution as suggested by Manning (1982).

A possible substitution could be of the type $\mathrm{AlNa}_{-1} \mathrm{Li}_{-1}$ as suggested by the trends roughly parallel to the exchange vectors

$$
\{X\}_{2} A l_{5} O_{13} N a_{-1} L i_{-1}(O H)_{-13}\{Y\}_{-4}
$$




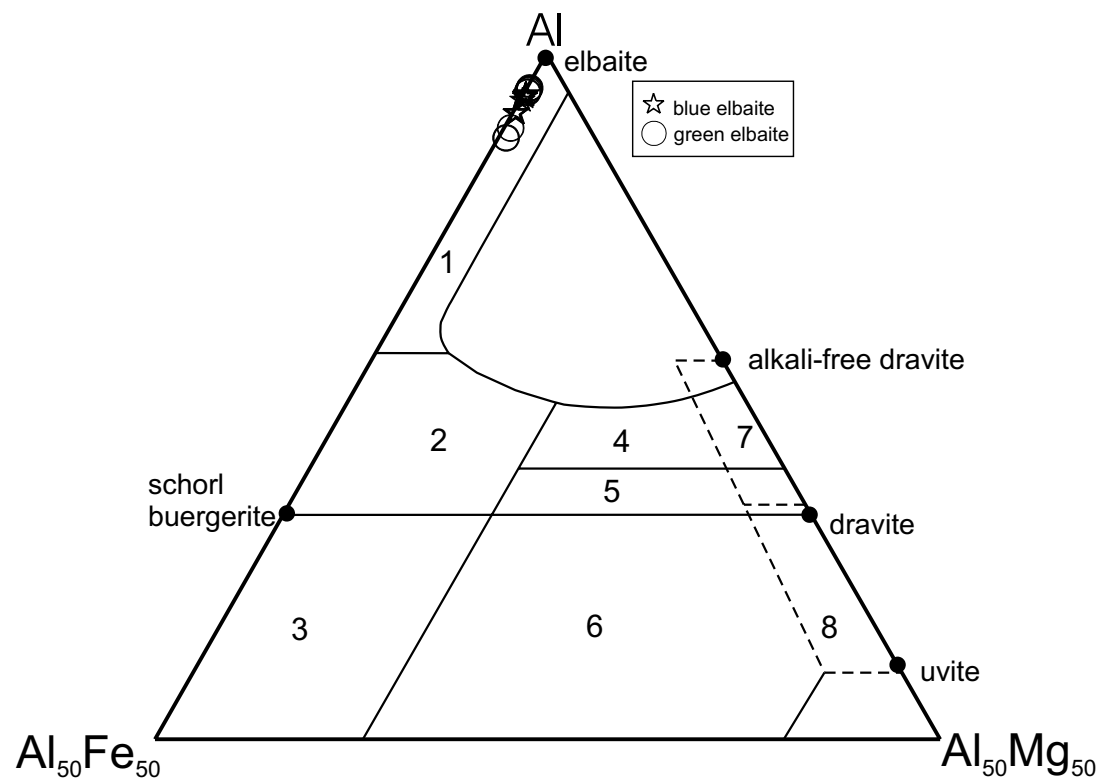

Fig. 3 - Al-Fe(t)-Mg diagram showing fields of the compositional range of tourmalines from different rock types proposed by Henry and Guidotti (1985). Field 1 is typical of tourmalines from $\mathrm{Li}$-rich granitoid pegmatites and aplites.

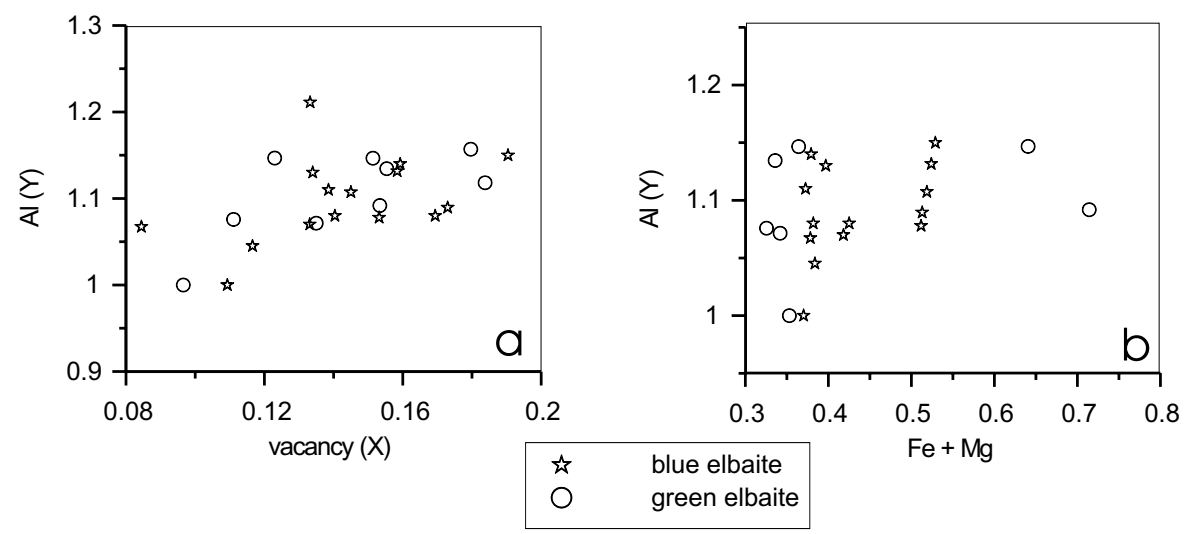

Fig. 4 - (a) $\mathrm{Al}$ in the $\mathrm{Y}$ site vs. vacancy in the $\mathrm{X}$ site of blue and green elbaites; (b) $\mathrm{Al}$ in the $\mathrm{Y}$ site vs. $(\mathrm{Fe}+\mathrm{Mg})$ of blue and green elbaites.

and

$$
\{X\} A l_{4.5} \mathrm{O}_{12} \mathrm{Na}_{-1} \mathrm{Li}_{-0.5}(\mathrm{OH})_{-12}\{Y\}_{-4}
$$

(Fig.5b), and slightly negative correlation between $\mathrm{Al}(\mathrm{Y})$ and $(\mathrm{Na}+\mathrm{Li})$. Substitutions that occur in the studied elbaites seem to be more complex than those reported in the literature and it will remain to be better explained.
All samples have high $\mathrm{FeO}(\mathrm{t})$, up to $3.96 \mathrm{wt} \%$ in the blue tourmalines, and up to $5.26 \mathrm{wt} \%$ in the green ones. Blue elbaites tend to have higher $\mathrm{Li}$ and $\mathrm{Mn}$ contents than the green ones (Fig. 6a, b). MnO contents $>1.5 \mathrm{wt} \%$ in tourmalines are relatively rare and usually associated to Na- and Li-bearing pegmatites, in elbaite-schorl tourmalines (Slivko 1961, 

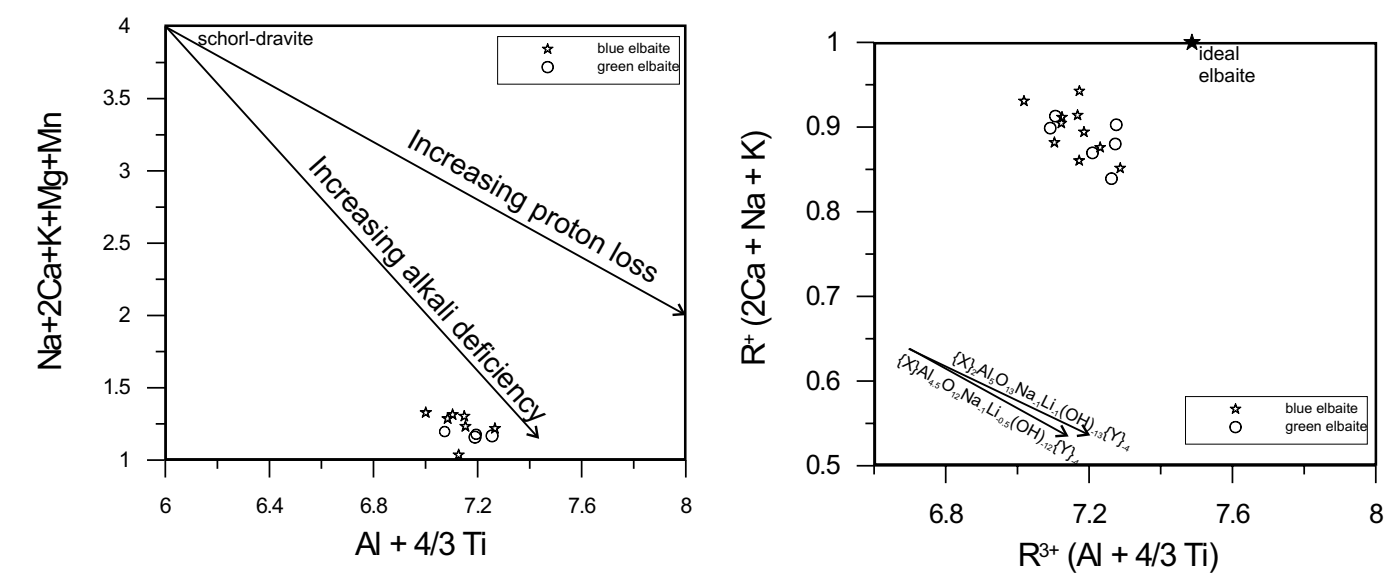

Fig. $5-$ (a) $\mathrm{R}^{3+}(\mathrm{Al}+4 / 3 \mathrm{Ti})$ vs. $\left(\mathrm{R}^{+}+\mathrm{R}^{2+}\right)(\mathrm{Na}+2 \mathrm{Ca}+\mathrm{K}+\mathrm{Mg}+\mathrm{Mn})$ diagram for elbaites of the Alto Quixaba pegmatite. Substitution trends are from Gallagher (1988). (b) $R^{3+}(\mathrm{Al}+4 / 3 \mathrm{Ti}) \mathrm{vs}$. $\mathrm{R}^{+}(\mathrm{Na}+2 \mathrm{Ca}+\mathrm{K})$ diagram for elbaites of the Alto Quixaba pegmatite. Substitutions trends are from Gallagher (1988).

Leckebusch 1978, Sahama et al. 1979). Mn contents in the studied tourmalines are high $(0.24-$ $0.42 \mathrm{apfu} ; 1.69-2.87 \% \mathrm{MnO}$, except for one sample that presents $\mathrm{MnO}=0,9 \%$ ) compared with average contents for blue elbaites, which usually present $\mathrm{Mn}$ contents $<0.30$ apfu (Selway et al. 1999). Shigley et al. (1986) described tourmalines with up to $8.86 \%$ $\mathrm{MnO}$, Gonzalez-Carreño et al. (1988) reported elbaites with up to 6,79\% MnO and Burns et al. (1994) with $\mathrm{MnO}$ values up to $6.23 \%$. Castañeda et al. (2003) reports tourmalines of the schorl-elbaite series from granitic pegmatites from Minas Gerais State, southeast Brazil, in which $\mathrm{MnO}$ is also high, up to $2.32 \mathrm{wt} \%$.

The studied elbaites are enriched in $\mathrm{Zn}$, up to $2.98 \mathrm{wt} \%$, a rather high value not commonly reported for other elbaites elsewhere. High $\mathrm{ZnO}$ values are reported for other Brazilian elbaites such as dark blue elbaites from other pegmatites of the Seridó region up to $2.43 \mathrm{wt} \%$ (D.R. Soares, unpublished data), and from Mina do Cruzeiro pegmatite, State of Minas Gerais, southeast Brazil, in which $\mathrm{ZnO}$ content is up to $2.0 \mathrm{wt} \%$ (J.C. Mendes, unpublished data).
Studied blue elbaites tend to present higher $\mathrm{Cu}$, $\mathrm{Ni}, \mathrm{Li}$ and Mn contents than the green ones (Fig. 6a through 6d). Semiquantitative X-ray fluorescence chemical analyses from five tourmalines of different colors indicate $\mathrm{TiO}_{2}(0.1 \%)$ in the dark green elbaite, and $\mathrm{Bi}_{2} \mathrm{O}_{3}$ (from 0.01 to $0.04 \%$ ) in all but in the blue elbaite (A.C.M. Ferreira, unpublished data).

\section{DISCUSSION AND CONCLUDING REMARKS}

Three distinct zones occur in the Alto Quixaba pegmatite, recognized on the basis of mineral assemblage and texture (external, intermediate and core), and a well-developed alteration zone in which one finds among other gem minerals, elbaites of excellent gem-quality of variable blue and green colors and hues. Refractive indexes, birefringence, relative density and parameters of the unit cell for these elbaites are similar to those reported for elbaites elsewhere. These are Mn- and Zn-rich elbaites that present vacancies in the $\mathrm{X}$ site from 12 to $17 \%$.

Complex and differentiated pegmatites present tourmalines from the external zones richer in $\mathrm{Fe}$ than those from the inner zone, in which tourmalines have much higher $\mathrm{Li}$ and $\mathrm{Al}$ contents (e.g. 


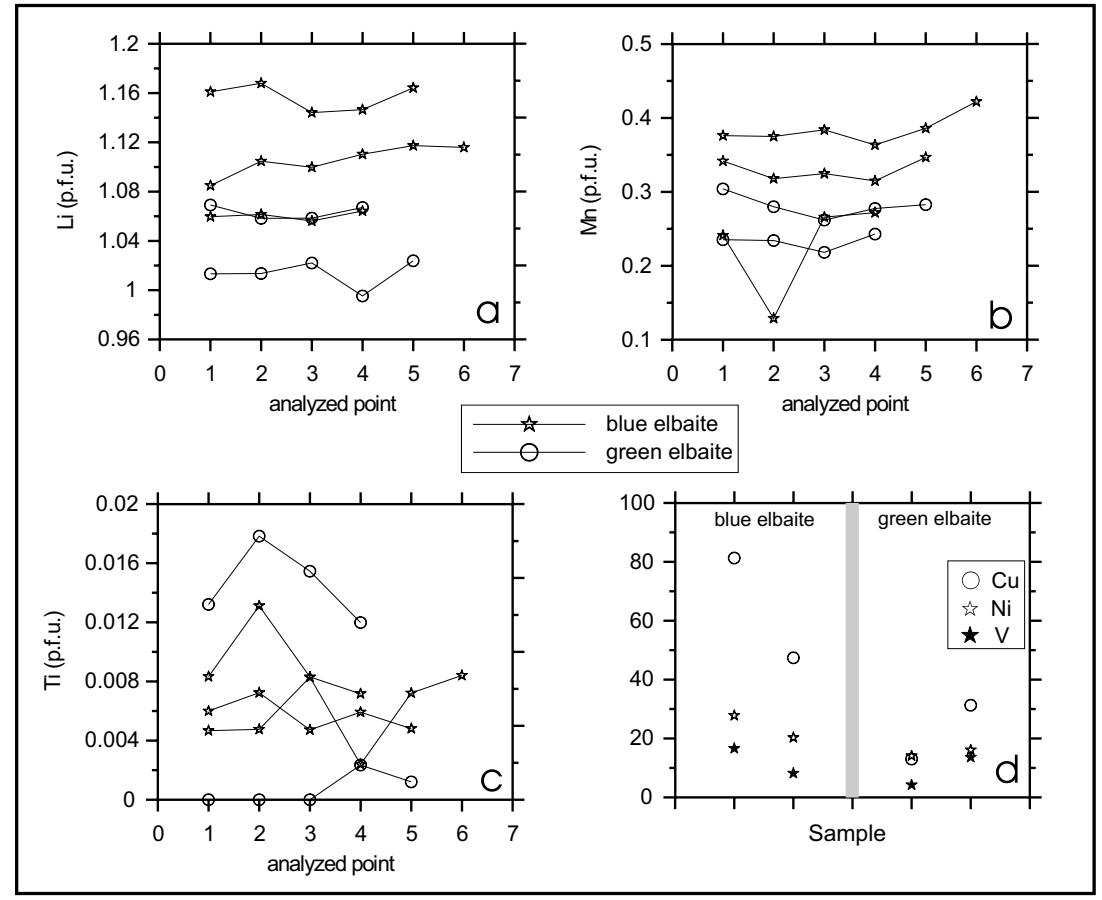

Fig. 6 - Compositional variations of studied blue and green elbaites. (a) through (c): different analyzed points in single grains; $\mathrm{Li}, \mathrm{Mn}, \mathrm{Ti}$, and $\mathrm{Fe}$ obtained by electron microprobe; (d) whole rock; $\mathrm{Cu}, \mathrm{Ni}$ and $\mathrm{V}$ obtained by ICP-AES.

Foord 1977, Jolliff et al. 1986). The Alto Quixaba pegmatite presents similar behavior, as the tourmaline from the external zone is schorl in which $\mathrm{FeO}$ contents vary from 12.5 to $13.8 \mathrm{wt} \%$ (A.C.M. Ferreira, unpublished data), while the blue and green elbaites from the replacement body in the central zone present $\mathrm{FeO}$ contents $<5.3 \%$, and $\mathrm{Li}_{2} \mathrm{O}$ varies from 1.59 to $1.81 \%$.

These Li-bearing tourmalines have relatively high Mn contents, in accordance with data reported in the literature (e.g. Sahama et al. 1979), although Mn-rich and Li-poor tourmalines also exist (e.g. Ayuso and Brown 1984). As observed by Dietrich (1985) elbaites contain larger trace element contents than do other tourmalines, probably because the former occur in complex pegmatites. Therefore some trace elements can have higher contents in some tourmalines, such as $\mathrm{Zn}$ as observed in the studied elbaites. Generally $\mathrm{Zn}$ is more common in green and blue elbaites than in red and yellow ones.
The concentration of major and/or trace elements, besides structural defects (color centers), charge transfer, or a combination of these parameters have been suggested as responsible for the different colors of tourmalines (e.g. Nassau 1978, 1983). On the basis of the concentrations of some known coloring agents such as $\mathrm{Fe}, \mathrm{Ti}, \mathrm{Mg}, \mathrm{Mn}, \mathrm{Cu}$, $\mathrm{Cr}, \mathrm{V}$, one can infer the possible origin of different colors of tourmalines. Yellowish green to bluegreen colors of elbaites from Mina da Batalha pegmatite, in the Borborema pegmatitic province, is attributed to $\mathrm{Cu}^{2+}$, while violet-blue and violet hues are due to Mn (Rossman et al. 1991). The studied elbaites could have their color due to $\mathrm{Cu}$ and $\mathrm{Mn}$ concentrations, which are higher in the blue elbaites compared to the green ones. Although not a coloring agent, it should be noted that Li contents is also higher in the blue elbaites than in the green ones.

Trace elements, however, can be or not responsible for, or even have some correlation with the 
color of tourmalines. Further studies (e.g. absorption spectroscopy) can shed some light on the origin of the different colors and hues of these elbaites.

\section{ACKNOWLEDGMENTS}

The authors are thankful to Prof. Dr. Bilal Essaid for the chemical analyses of the elbaites by Inductively Coupled Plasma - Atomic Emission Spectrometry performed at the Saint-Etienne School of Mines, France.

\section{RESUMO}

O pegmatito Alto Quixaba na região do Seridó, nordeste do Brasil, é um corpo subvertical de direção $60^{\circ} / 80^{\circ} \mathrm{SW}$ intrudindo discordante biotita xistos da Formação Seridó. Apresenta três zonas distintas em termos de mineralogia e textura, além de uma zona de alteração em forma de dique na qual ocorre, entre outros minerais-gema, elbaítas coloridas. As elbaítas ocorrem como cristais prismáticos alongados de acordo com o eixo $\mathrm{C}$, com faces arredondadas e estrias paralelas a esse eixo. Os cristais são uniaxiais negativos e apresentam forte pleocroísmo; índices de refração $\mathrm{n}_{\mathrm{E}}=1,619-1,622$ e $\mathrm{n}_{\mathrm{O}}=1.639-$ 1.643 , birrefrigência entre 0,019 e 0,021 , densidade relativa de 3,07 , e os parâmetros seguintes da célula unitária: $a \mathrm{o}=15,845 \AA$, $c \mathrm{o}=7,085 \AA \mathrm{e} \mathrm{V}=1540,476 \AA$ ․ O sítio $\mathrm{X}$ apresenta deficiência em álcalis entre 12 e 17\%. As elbaítas são relativamente ricas em $\mathrm{MnO}$ (1,69 a 2,87\%) e $\mathrm{ZnO}$ (até 2,98\%).

Palavras-chave: elbaíta, turmalina, província pegmatítica do Seridó.

\section{REFERENCES}

Almeida FFM, Hasui Y, BRito Neves BB AND FUCK RA. 1981. Brazilian structural provinces: an introduction. Earth Science 17: 1-9.

ANDERSON BM. 1980. Gem testing, Butterworths \& Co Ltda, $9^{\text {th }}$ ed., London, 434 p.

AYuso RA AND BROWn CE. 1984. Manganese-rich red tourmaline from the Fowler talc Belt, New York. Can Mineral 22: 327-331.

Benvenuti M, Costagliola P, Lattanzi P And TONELLI G. 1991. Mineral chemistry of tourmalines from Bottino mining district, Apuane Alps (Italy). Eur J Mineral 3: 537-548.

Brandstaetter F AND Niedermayr G. 1994. Inclusions of native copper and tenorite in cuprianelbaite tourmaline from Paraíba, Brazil. Gems and Gemology 30: 173-183.

Burns PC, MaCDonald DJ AND Hawthorne FC. 1994. The crystal chemistry of manganese-bearing elbaite. Can Mineral 32: 31-41.

BURT DM. 1989. Vector representation of tourmaline compositions. Amer Mineral 74: 826-839.

CAmeron EN, Jahns RH, McNair A And PAGE LK. 1949. Internal structure of granitic pegmatites. Econ Geol Monograph 2: 115.

Castañeda C, Eecckhout SG, Grave E, Botelho NF AND SoARes ACP. 2003. Fenômeno de ordem-desordem local em turmalinas naturais e tratadas da série schorlita-elbaíta. Rev Bras Geocienc 33: 75-82.

Deer WA, Howie RA and Zussman J. 1966. An introduction to the rock-forming minerals. Logman Group Ltd, 528 p.

DIETRICH RV. 1985. The tourmaline group. Van Nostrand Reinhold Company, New York, 300 p.

DONNAY G AND BARTON R. 1972. Refinement of the crystal structure of elbaite and the mechanism of tourmaline solid solution. Tschermaks Min Petr Mitt 18: $273-286$.

FoIt FF And Rosenberg PF. 1977. Coupled substitutions in the tourmaline group. Contr Mineral Petrol 62: 109-127.

FoIt FF And Rosenberg PF. 1979. The structure of vanadium-bearing tourmaline and its implications regarding tourmaline solid-solutions. Amer Mineral 64: 788-798.

Foord EE. 1977. The Himalaya dike system, Mesa Grande district, San Diego Country, California. Min Recor 8: 475-478.

Gallagher V. 1988. Coupled substitutions in schorldravite tourmaline: new evidence from SE Ireland. Mineral Mag 52: 637-650.

Gonzalez-CARreño T, Fernández M And SAnZ J. 1988. Infrared and electrón microprobe análisis of tourmalines. Phys Chem Minerals 15: 452-460.

GÜBELIN E. 1979. Internal world of gemstones - documents from space and time. Butterworts \& Co Ltd., $2^{\text {nd }}$. ed., London, p. 188-189. 
Guilbert JM AND PARK JR CF. 1986. The geology of ore deposits. W.H. Freeman and Company, New York, $985 \mathrm{p}$.

HAWTHORNE FC. 1996. Structural mechanisms for light-element variations in tourmaline. Can Mineral 34: 123-132.

HenRy DJ AND GuidotTi CV. 1985. Tourmaline as a petrogenetic indicator mineral. An example from the estaurolite-grade metapelites of MW Maine. Amer Mineral 70: 1-15.

Jolliff BL, Papike JJ AND Shearer CK. 1986. Tourmalines as a recorder of pegmatite evolution: Bob Ingersoll pegmatite, Black Hills, South Dakota. Amer Mineral 71: 472-500.

LeCKebusCH R. 1978. Chemical composition and colour of tourmaline from Dane Pech (Nuristan, Afghanistan). Neus Jahrb Mineral Abhan 133: 5370.

MacDonald DJ And Hawthorne FC. 1995. The crystal chemistry of $\mathrm{Si} \leftrightarrow \mathrm{Al}$ substitution in tourmaline. Can Mineral 33: 849-858.

MANNING DAC. 1982. Chemical and morphological variation in tourmalines from the Hub Kapong batholith of peninsular Thailand. Mineral Mag 45: 139-147.

NASSAU K. 1978. The origin of color in minerals. Amer Mineral 63: 219-229.

NASSAU K. 1983. The physics and chemistry of color: the fifteen causes of color. J Wiley \& Sons, New York, USA.

NORTON JJ. 1983. Sequence of mineral assemblages in differentiated granitic pegmatites. Econ Geol 78: 854-874.

PaIVA G. 1946. Províncias Pegmatíticas do Brasil. DNPM/DFPM, Rio de Janeiro, RJ, Brasil, Boletim 78: 13-21.
POVONDRA P. 1981. The crystal chemistry of tourmalines of the schorl-dravite series. Acta Univ Carol Geol 3: 223-264.

READ PG. 1982. Dictionary of gemology, Butterworth and Co Ltd, London, $240 \mathrm{p}$.

Rossman GR, Fritsch E AND Shigley JE. 1991. Origin of color in curprian elbaite from São José da Batalha, Paraíba, Brazil. Amer Mineral 76: 14791484.

SAHAMA THG, VON KNORRING O AND TORNROOS R. 1979. On tourmalines. Lithos 12: 109-114.

Selway JB, Novak M, Cerny P and Hawthorne FC. 1999. Compositional evolution of tourmaline inlepidolite-subtype pegmatites. Eur J Mineral 11: 569-584.

Shigley JE, KAne RE And MAnson DV. 1986. A notable Mn-rich gem elbaite tourmaline and its relationship to "tsilaisite". Amer Mineral 71: 12141216.

SILVA MRR DA. 1995. Borborema pegmatitic province: geological and geochemical characteristics. Jour South Amer Earth Sci 8: 355-364.

SLIVKO MM. 1961. On manganese tourmalines. Intern Geol Rev 3: 195-201.

SoARES DR, FERreira ACM, Rodrigues DA SILVA R AND FERREIRA VP. 2000. Alkali-deficient elbaite from pegmatites of Seridó region, Borborema province, NE Brazil. Rev Bras Geocienc 30: 293296.

VilarRoel-Leo HS. 1971. Método de Ito para indexado de diagramas de policristal. Universidade Austral do Chile/ Inst. de Biofísica, Vadivia, Chile, p. 3-8.

WERDING G AND SCHREYER W. 1984. Alkali-free tourmaline in the system $\mathrm{MgO}-\mathrm{Al}_{2} \mathrm{O}_{3}-\mathrm{B}_{2} \mathrm{O}_{3}-\mathrm{SiO}_{2}$ $\mathrm{H}_{2} \mathrm{O}$. Geochim Cosmochim Acta 48: 1331-1344. 